\title{
Racionalidad y normatividad en el conocimiento científico
}

\author{
AMPARO GÓMEZ \\ Universidad de La Laguna
}

\section{Introducción}

Una de las controversias más importantes sobre la ciencia y su desarrollo es la que tiene lugar en las dos últimas décadas entre la sociología del conocimiento y la filosofía de la ciencia. Esta controversia enfrenta a los sociologos del conocimiento empeñados en reducir el conocimiento científico a lo sociológico y a los filósofos de la ciencia que niegan la viabilidad de tal empeño. El debate que se genera es enormemente complejo dada la variedad de temas que intervienen en la discusion: cuestiones de tipo metodológico, epistemológico y axiológico; interpretaciones del cambio y progreso científico; teorías de la racionalidad; teorias de la explicación sociológica; amén de las cuestiones propias de la sociología de la ciencia.'

Ofrecer una breve panorámica de este debate es, por tanto, difícil; en el trabajo que a continuación desarrollo me limitaré a presentar un esbozo de la polémica entre la versión más fuerte de la sociología del conocimiento (el Strong Programme) y la filosofía de la ciencia racionalista que ha debatido con aquella en términos de alguna teoría de la racionalidad científica. El objetivo central que se persigue es ofrecer una panoramica de las tesis racionalistas y su evolución en el debate con el Strong Programme; por tanto, al mostrar una de las partes en litigio, ello nos obligará a tener en cuenta, sólo de forma muy esquenática, a la otra. En cualquier caso las tesis sociologistas quedarán perfectamente recogidas en otro trabajo que se incluye en este mismo número de Isegoria.

Comenzaré con un breve planteamiento histórico de la cuestión para centrarme a continuación en el enfoque racionalista exponiendo tanto la versión normativa fuerte como la descriptiva más moderada.

\section{La sociología}

Los filósofós de la ciencia tradicionalmente han propuesto reconstrucciones racionales del conocimiento científico. La reflexión sobre la ciencia, sus teorías, procedimientos de justificación y desarrollo, se ha planteado en términos de los factores intrínsecos característicos de la ciencia y su práctica. Los elementos externos se desechan del análisis y son considerados objeto de estudio de otras disciplinas como la historia o la sociologia, nunca de la filosofía interesada en la cuestión lógica y metodológica de la validez, según la conocida afirmación de Popper. ${ }^{2}$

La distinción entre factores internos y externos, incuestionable para los filosofos de la ciencia clásicos, fue admitida también por los primeros sociólogos como Merton ${ }^{3}$ o Mannheim. ${ }^{4}$ Este áltimo, aunque fue el primero en proponer una sociología del conocimiento propiamente dicha, aceptó la distinción para las ciencias naturales pero no para las culturales. ${ }^{5}$ La sociologia clásica coincide, pues, con la filosofía 
en señalar un ámbito propio para la sociología y otro para la filosofía de la ciencia.

Esta situación se va modificando paulatinamente. A ello contribuyen ciertos desarrollos que se dan en el seno de la misma filosofia de la ciencia. Así, la idea de que el conocimiento científico ha de ser estudiado en terminos de su reconstrucción según los criterios logicistas y empiristas del Positivismo Lógico, se resquebraja a partir de los años cincuenta-sesenta. Esto ocurre en dos frentes: uno, el abierto por los trabajos de Quine, Duhem, o Hesse en torno a la posibilidad de una epistemología naturalizada; otro; el constituido por las aportaciones críticas de autores como Hanson, Kuhn o Feyerabend ( $\sin$ olvidar el papel que jugó Popper en el cuestionamiento de ciertas tesis del Circulo de Viena).

En ninguno de los dos frentes, sin embargo, se pretende sustituir la epistemología por la sociología del conocimiento. La sociologia complementaría a la epistemología manteniendo ésta su independencia y capacidad explicativa, Con Kuhn, por ejemplo, las reconstrucciones racionales de la ciencia no se abandonan, aunque éstas hayan de cobrar otro carácter.

Scrá la sociología del conocimiento de la década de los setenta la que cuestionará frontalmente las reconstrucciones racionales de la ciencia y su exclusión de los factores externos. El éxito de las investigaciones sociológicas en esta década y el optimismo que desencadena llevan a la propuesta de un programa sociologista enormemente ambicioso. Se afirma que la sociología del conocimiento, al modo de las ciencias duras, puede constituirse en una teoria capaz de elaborar explicaciones causales de los contenidos cientificos, de las creencias cientificas, a través de leyes sociales empiricas y contrastables, sin interesarse por la verdad, falsedad, racionalidad o irracionalidad de tales creencias según la tesis de la simetria mantenida por Bloor. ${ }^{7}$ No sólo se niega la distinción externo-interno sino que afirma la prioridad de los factores externos en la explicación con la consiguiente eliminación de los internos.

La sociología de la ciencia es entendida como una teoría general y un programa de investigación, es decir, como una ciencia de la ciencia. ${ }^{8}$ Esto es lo que se conoce como Strong Programme en los términos en que lo formulan Barnes $^{9}$ y Bloor ${ }^{\text {th }}$ Sería la sociología del conocimiento la que podria ofrecer una verdadera explicación cientrfica de la ciencia.

\section{La racionalidad}

Desde la filosofía de la ciencia se rechazan las tesis del Strong Programme cuestionadoras de las posiciones racionalistas. El debate en torno al papel de los factores externos e internos en la ciencia se centra en una cuestion fundamental: $¡$ son las creencias cientificas mero resultado causal de factores sociológicos o producto de criterios evaluativos internos en cuyos términos se las establece y acepta?

Los racionalistas, desde luego, mantienen lo segundo. La argumentación esgrimida como fundamento de esta opción varía desde posiciones fuertemente normativas hasta posiciones más moderadas de corte descriptivo. Racionalistas como Popper, Lakatos o Laudan coinciden, con ciertos matices, en una tesis común: las creencias científicas son producto de la práctica científica guiada por criterios epistemológicos, metodológicos y según metas y fines científicos objetivamente racionales y universalmente establecidos.

En este sentido, Popper"l sostiene, 
como es bien sabido, que la ciencia se basa en el método crítico en función del cual se establecen teorías cada vez más capaces en la resolución de problemas y cada vez más próximas a la verdad. Esto sería lo característico de la racionalidad científica. Popper, sin embargo, en una de sus últimas obras admite la existencia de intereses extracientíficos en torno a la ciencia y la dificultad de eliminarlos. ${ }^{12}$ No obstante, lo característico del conocimiento científico sigue siendo "[...] la distinción entre intereses que no pertenecen a la búsqueda de la verdad y el interés puramente científico por la verdad" ${ }^{13}$ Una "de las tareas permanentes de la critica cientifican y la discusión científica consiste en evitar la confusión de intereses y en eliminar las valoraciones extracientíficas del ámbito de las cuestiones de verdad. A largo plazo, factores como el medio social o ideológico del investigador tienden a eliminarse filtrados por la crítica.14

Lakatos, 15 a pesar de su critica a Popper, afirma que el fundamento del conocimiento cientffico se basa en la reconstrucción racional de la historia interna de la ciencia. ${ }^{16}$ La historia externa consiste en aquello que los científicos hacen desviado de las reglas metodologicas derivadas de la filosofia de la ciencia. Esta conducta y sólo ésta es explicada por causas sociologicas o psicológicas. Asi las leyes inmanentes por las que se rige el conocimiento son leyes de la racionalidad.

Una teoría es racional (y por tanto sus reglas y conducta científica) si permite predecir hechos nuevos. Ello permite distinguir entre programas de investigación progresivos y degenerativos: la historia interna de los programas de investigación muestra su progreso. La controversia científica se resuelve racionalmente si los científicos cambian de un programa degenerativo a uno progresivo como habitualmente ocurre según Lakatos. ${ }^{17}$

A la pregunta: ccuándo ha de abandonar el cientifico racional un programa?, Lakatos ${ }^{18}$ responde modificando su definición de racionalidad. La racionalidad no reside tanto en lo que se haga, sino en cómo se haga, lo que tiene que ver con cuestiones como la sinceridad $u$ honestidad en el abandono del programa. ${ }^{19}$ Hay aquí un cierto desplazamiento hacia lo subjetivo, pues es el científico honesto (se equivoque o no) el que decide abandonar un programa por considerarlo degenerativo, $y$ al hacer esto procede racionalmente. Ello implica una enorme cantidad de cuestiones, entre otras que si un científico no abandona un programa degenerativo porque honestamente considera que no hay razones para ello tal comportamiento es racional. Aparece así una dualidad en el corazón de la concepción de Lalkatos. ${ }^{20}$

Laudan $^{2 t}$ establece con nitidez cuál es el dominio de la explicación racional y cuál el de la sociologica. ${ }^{22}$ La pregunta fundamental para él es: "¿qué tipo de creencias científicas son susceptibles de un análisis sociológico, y cuáles no lo son, es decir qué creencias se explican en términos racionales y cuáles en términos sociológicos?».

La respuesta siguiendo a Mannheim se da en los siguientes términos: son las creencias no inmanentes, es decir, aquellas que no están racional y naturalmente derivadas o fundadas en otras, las que han de ser tratadas en términos sociológicos. ${ }^{23}$ Si una creencia se relaciona racionalmente con otra, de la que se sigue de forma natural, esto es lo que la explica puesto que ésta es la razón de que el cientifico la acepte, siempre que una creencia pueda ser explicada por razones adecuadas no hay necesidad de buscar explicación alternativa en términos de causas sociales. ${ }^{24}$ 
Laudan es, sin embargo, perfectamente consciente de que el sociologo del conocimiento no acepta la distinción, pues, según éste, todas las creencias son explicables sociologicamente, se deriven racionalmente o no. Buena parte de la argumentación que desarrolla Laudan en contra de la sociología cognitiva está dirigida a demostrar que esta tesis es insostenible y que, por tanto, la sociología ha de limitarse a las explicaciones no racionales de las creencias.

Así, y en primer lugar, si la tesis del sociólogo fuese cierta éste no podría argumentar a favor de la plausibilidad de la misma, como de hecho hace, ya que al actuar así esgrime razones evaluativas no sociológicas, con lo cual la sociología del conocimiento se postula, de hecho, en terminos racionalistas.

Por otro lado, Laudan pone el peso de la prueba en el lado del sociólogo pues éste ha de responder a la siguiente pregunta: ¿qué añade la explicación sociológica a la explicación racional? Laudan mantiene que nada, pues toda explicación ha de dar cuenta de las condiciones suficientes y ucon tal que podamos mostrar que el antecedente más crucial y relevante para la aceptación de la creencia fue un proceso de razonamiento bien fundado por parte del portador de la creencia, ya disponemos de tales condiciones ${ }_{2}{ }^{25} \mathrm{El}$ sociólogo es incapaz de ofrecer una sola explicación en términos de condiciones suficientes, la explicación sociologica es insostenible (Laudan se apoya aquí en S. Mill y su concepción de la explicacion) ${ }^{26}$

En la explicación de la elección entre teorias Laudan considera que siempre que un científico no elija la teoría más progresiva o la tradición más adecuada en términos de su capacidad de resolución de problemas hemos de recurrir al sociólogo (o psicólogo) para su comprensión, puesto que no hay posibilidad de una explicación racional. Mientras esto no ocurra basta con la explicación racional. ${ }^{27}$

La respuesta fuertemente racionalista de estos autores ha de afrontar una crítica fundamental: la acusación de que no da cuenta del proceso real por el que los cientificos establecen y aceptan creencias cientificas o eligen entre teorias rivales. Ofrece una evaluación de la ciencia y su desarrollo pero no una explicación de lo ocurrido en los términos que sucedió Los modelos racionalistas no explicarían el caso en que el científico no acepta la creencia o elige la teoria que el modelo afirma como superior. Tampoco explicarian cuando el científico lo hace pero por razones diferentes a las que el modelo da. En ambos casos, según los autores examinados, sería precisamente la sociología la encargada de la explicación, con lo que gran parte de la reconstrucción de la ciencia quedaría en manos de la sociología del conocimiento ${ }^{28}$

Esto lleva a una serie de autores a rebajar el elemento normativo en los modelos de reconstrucción racional restringiendo las exigencias y tratando de aproximarse a las condiciones reales de la práctica científica. Sin embargo, estos desarrollos logran su objetivo sólo parcialmente, al quedar en un terreno intermedio entre lo prescriptivo $y$ lo descriptivo. Autores como J. Agassi, ${ }^{24}$ R. Wettersten, ${ }^{30}$ J. Kekes ${ }^{31}$ o I.C. Jarvie ${ }^{32}$ en buena medida restringen el concepto de racionalidad de Popper oscilando entre una racionalidad subjetiva y el mantenimiento de criterios objetivos como el principio de resolución de problemas. Este principio se entiende como un componente intrínseco al comportamiento racional real, pero también como un componente del conocimiento científico y su desarrollo, en cuyos términos es evaluable. La resolu- 
ción de problemas permite describir, pero también medir, la racionalidad y el progreso científico en términos del progreso de los mismos problemas y el progreso en la elección de problemas cientificos. En la práctica humana la situación es la misma, con lo que se afirma el progreso incluso ético en términos del progreso en los problemas éticos y su elección. ${ }^{33}$

En línea con esta posición intermedia se puede interpretar también el trabajo de Laudan Science and Values, en el que modifica el componente fuertemente normativo de su obra anterior presentando un modelo reticular de justificación que se pretende descriptivo sin eliminar totalmente el componente normativo. ${ }^{34}$ Cree que se puede evaluar racional y críticamente objetivos y metas científicas; que es posible una crítica axiológica. La indagación axiológica se sitúa en un terreno extrano para la filosofía de la ciencia, no pertenece a la filosofia prescriptiva y tampoco a la descriptiva. Sin embargo, Laudan mantiene que la discusión axiologica puede establecer como más adecuados unos valores que otros y permitir interpretar en estos términos el cambio y progreso racional de la ciencia.

\section{La racionalidad descriptiva}

El interés por formular un modelo de explicación racional que evite las críticas señaladas a las reconstrucciones normativas de la ciencia da lugar a una propuesta racionalista claramente descriptiva que no se limita a evaluar desde principios prescriptivos lo que ocurrio, sino que trata de explicar por qué ocurrió. ${ }^{35}$ Se reconoce el problema de la normatividad al mismo tiempo que se afirma que la explicación racional es imprescindible para hacer inteligible a la ciencia y su desarrollo.
Tal propuesta se basa en la concepción de la racionalidad (acción y olección racional) que desarrollan autores como P. Suppes, ${ }^{36}$ Elster, ${ }^{37}$ Cherniak, ${ }^{38}$ entre otros, y que ha sido aplicada a la ciencia, con matices diferentes, ${ }^{39}$ por Newton-Smith, Brown, MacMullin, o G. Gutting.40 Se configura así un programa racionalista que rebaja las exigencias de los programas fuertes y que se sitúa explícitamente en el ámbito de la racionalidad instrumental.41

Lo que postula tal programa es que a la pregunta epor qué ocurrió lo que ocurrió, se responde en función de las razones antecedentes de personas que actúan en términos, no de una racionalidad normativa, sino de una racionalidad descriptiva "mínimas, 42 cimperfecta», "problemática» ${ }^{43}$ según la terminologia de cada autor. ${ }^{44}$ La idea básica, compartida por estos autores, es que a pesar de las críticas a las reconstrucciones racionalistas fuertes la noción de racionalidad es clave en la explicación del conocimiento científico. Si se la elimina no habrá forma de decidir entre propaganda y argumento racional, ${ }^{45}$ con lo que el sociólogo mismo no podría siquicra comenzar su labor pues no podría identificar las creencias científicas que pretende explicar y desde luego tampoco podría explicarlas.

No podría hacerlo según NcwtonSmith porque, si el papel del sociólogo es explicar las creencias existentes y éstas existen porque los sujetos las han considerado verdaderas según criterios de evaluación diferenciales, entonces hay que atender a tal evaluación para dar cuenta de por qué las mantienen (o rechazan). La sociología al explicar por qué se sostienen tales creencias está obligada a incluir estos criterios evaluativos. ${ }^{46}$

D. Bloor ${ }^{47}$ y B. Barnes ${ }^{48}$ afirman la indeseabilidad metodológica de utilizar la evaluación diferencial de creencias 
en la explicación. Admiten que es cierto que evaluamos diferencialmente las creencias como racionales o no, pero esto no ha de ser tenido en cuenta por el sociólogo para su explicación.

Newton-Smith ${ }^{4 y}$ responde a esta cuestion mostrando que una explicación causal no puede obviar este hecho. Supongamos, dice, una creencia perceptualmente verdadera y una creencia perceptualmente falsa. En ambos casos la explicación cs causal, pero en un caso el estado de cosas que debe mencionarse en la explicación de por qué se acepta esa creencia, le da valor de verdad. En el otro, tal estado de cosas no ha de incluirse en la explicación de por qué se mantiene la creencia, puesto que no tiene nada que ver en ello. El estado de cosas que hace que su creencia sea falsa no es causa de que tenga esa creencia. Por tanto no podemos empezar a decidir qué clase de explicación es apropiada hasta que sepamos si la creencia es verdadera o no. En ambos ejemplos la explicación es causal, pero en el caso de una creencia perceptual verídica la cadena causal involucrada atraviesa el estado de cosas que da a la creencia su valor de verdad incluyendo los criterios evaluativos utilizados por el sujeto. ${ }^{50}$

Dos cosas se pueden objetar a Newton-Smith todavia: que se mantienen creencias en términos evaluativamente plurales, no sólo por su verdad, y además, que cuando se mantienen creencias falsas éstas no están exentas de una explicación que recoja la evaluación diferencial y su aplicación por el sujeto.

Su análisis puede ampliarse a los dos casos. Sencillamente en el primero los criterios de evaluación no tienen que estar sólo relacionados con la verdad, sino con otros valores epistemológicos que se incluirían en el estado de cosas que explican racionalmente la creencia. En el segundo la cadena causal contempla el estado de cosas que da a la creencia su valor de verdad para el sujeto, incluyendo su evaluación diferencial (aunque ésta le lleve a sostener una creencia falsa según criterios epistemologicos objetivos) ${ }^{5 !}$

En otros términos, lo que interesa para la explicación es cómo evaluó la situacion el sujeto, ya que en función de ello sostiene su creencia (sea esta valorada como verdadera o falsa desde criterios objetivos). Esto es lo que la explica, lo que da razón de por qué se la sostiene, no importa que la consideremos razonable o no, la sepamos verdadera o no. ${ }^{5 z}$ Lo que interesa es que el tiene una razón mejor para creer en $p$ que en no $p$, o para suspender el juicio en lo que refiere a $p$. Proporcionar una explicación racional no exige más.

Todo lo que se afirma desde este modelo de racionalidad es que las personas actúan (eligen, deciden) racionalmente en la medida en que realizan la acción que estiman adecuada en su situación para lograr deteminados fines, según su evaluación de tal situación (incluyendo la evaluación de los medios disponibles). Una explicación racional consiste, pues, en especificar las razones antecedentes sabiendo que las personas generalmente son consecuentes y hacen lo que creen más adecuado en su situación, es decir, actúan según un principio de racionalidad mínima, que opera como generalización probabilistica en el modelo explicativo. ${ }^{53}$

Este modelo aplicado a la ciencia supone una explicación, descriptiva y contextualizada de las decisiones, elecciones y actuaciones que llevan a cabo los científicos de acuerdo con los criterios, normas y objetivos dominantes en la comunidad cientifica correspondiente.

También permite explicar por qué los sujetos actúan, a veces, de forma inadecuada según los criterios y normas dominantes en la comunidad. Esto ocurre por razones internas a la 
ciencia: sencillamente se interpreto mal la evidencia disponible, el científico aplico equivocadamente los criterios evaluativos, a no compartía tales criterios, por ejemplo. La explicación de lo ocurrido sigue siendo racional. No hay que olvidar que los científicos actúan o deciden en muchas ocasiones en condiciones de riesgo $\mathrm{y}$, sobre todo, de incertidumbre que dificultan enormemente su actuación (pueden no saber siquiera cuál es la probabilidad atribuible a cada resultado, lo que obliga a elegir según una función de utilidad esperada). En cualquier caso se ha actuado de forma racional, en función de criterios, normas, y objetivos puramente científicos, y su actuación es explicable en tales términos.

Por otro lado, puede ocurrir que se haya actuado en funcion de razones puramente externas a la ciencia: intereses, razones ideológicas o personales, por ejemplo. En este caso la explicación racional mínima es perfectamente adecuada en têrminos de tales razones externas evaluativamente establecidas desde criterios y objetivos también externos a la ciencia. Es decir, la existencia de factores externos que influyen en la ciencia no implica una explicación sociológica a modo del Strong Programme. ${ }^{\text {st }}$

El modelo racional expuesto permite dar cuenta de la elección entre teorias científicas. Lo que interesa son las razones a partir de las cuales los cientificos deciden a favor de una u otra teoría, en un momento histórico determinado. Tales razones pueden tener como resultado la elección de una teo. ria que posteriormente se considera objetivamente superior a su competidora, pero puede tener el resultado contrario. Esto no supone que tal resultado sea irracional, los cientificos procedieron racionalmente segün los criterios dominantes en su comunidad $y$ en tales términos es explicable su elección. Una cosa es que las razones que se aduzcan no coincidan con las razones que un modelo máximamente racional da del cambio, otra muy distinta, que estas razones no sean racionales, fruto del cálculo, la discusión o la argumentación por ejemplo.

No olvidemos que en situaciones de revolución y cambio cientifico las elecciones se dan en condiciones de incertidumbre. Se elige en términos de creencias y expectativas acerca de los resultados esperados de una teoría, que no pueden garantizarse de antemano. Una nueva teoría puede parecer la más adecuada de acuerdo con los criterios de evaluación dominantes en el momento $y$, sin embargo, demostrarse posteriormente como la peor (a al contrario). En el contexto de su adopción, dificilmente una teoria ha podido demostrar su superioridad objetiva de forma inequivoca.

Según este modelo, se puede explicar, por ejemplo, por qué planck optó por su ley de distribución de la radiación producida por un cuerpo negro, mediante una reconstrucción de las creencias desde las que Planck en su momento opta por esta opción. Una cosa son las razones de Planck en el

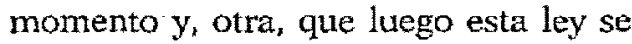
pueda valorar como un progreso objetivo, y haya razones para verlo asi; ambas cuestiones no tienen por qué coincidir. Un ejemplo de creencia metodológica general que se podría citar para explicar la aceptación de la teoría de Planck por la comunidad científica es la creencia en la importancia de la unificación teórica. Esta, en parte, motivó a la comunidad a preferir la formula única de Planck, que cubria todas las temperaturas, en lugar de la fórmula de Wien para bajas temperaturas y la de Rayleigh-Jeans para temperaturas altas. 
Finalmente resaltaremos una cuestión importante en relación al modelo de explicación racional mínima. Este modelo permite atender a un hecho poco considerado hasta el momento: que las decisiones y actuaciones cientificas se dan en contextos interactivos. ${ }^{55}$ Los científicos no son decisores o actores aislados, la ciencia es resultado de la práctica colectiva de comunidades, lo que implica contextos de interacción estratégica y comunicación. Por tanto, la comprensión de la ciencia y su desarrollo exige las aportaciones de teorías como las de la acción-elección colectiva y teoria de juegos, pero también teorias del acuerdo $y$ consenso, $y$ en consecuencia de la argumentación con sus formas de persuasión y retórica, como reconoce la misma filosofía de la ciencia actual. 56 Es decir, esto implica atender a las condiciones de la acción individual y colectiva, pero también a las condiciones de diálogo y comunicacion racional en el seno de las comunidades cientificas. Sin atender a tales aspectos la explicación de la ciencia es parcial.

\section{Conclustón}

A modo de conclusión señalaremos algunas de las implicaciones del modelo racional mínimo expuesto. Desde este modelo no se niega la influencia de factores sociales, económicos o políticos, en el conocimiento cientifico; lo que se rechaza es que esto ocurra en los términos que afirma el del Strong Programme eliminando la importancia de los factores internos y su papel fundamental en la explicación de los contenidos científicos. ${ }^{57}$ La empresa científica y su desarrollo se hace inteligible, sobre todo, en términos de tales factores internos. ${ }^{58}$
Por otro lado, se afirma que los actores proceden racionalmente en relacion a los factores internos pero también a los externos. En ambos casos, el tipo de explicación es la misma: racional mínima. ${ }^{5}$ No se trata de que cuando la explicación es interna se atienda a las razones del agente incluyendo su evaluación del estado de cosas pertinente, mientras que cuando es externa opere según leyes deterministas a modo de la mecánica clásica tal como pretende el Strong Programme. ${ }^{60}$

Una consecuencia que se deriva de este modelo de explicación es que no presupone un tipo de racionalidad cientifica diferente de la racionalidad que aplican los seres humanos en cualquier contexto de acción o decisión. El proceder racional es sustancialmente el mismo en el ámbito de la ciencia que fuera de $6 \mathrm{l}$, la diferencia estriba en las constricciones a que está sometida la práctica cientifica fuertemente normativizada, no en la naturaleza del proceder racional.

Por último es necesario mencionar que las fronteras de la controversia entre sociologistas y racionalistas se han ido modificando en el transcurso del debate. Los sociólogos de la ciencia han ido paulatinamente reconociendo la importancia de los factores internos y la necesidad de proponer una teoría de la racionalidad natural ( $\sin$ por ello abandonar la tesis del origen social de los contenidos científicos), mientras que los racionalistas han ido abandonando, como hemos visto, los requerimientos normativos y admitiendo la importancia de los factores externos en ciertos términos. En cualquier caso el tema sigue abierto y es objeto de atencion por parte de filosofos y sociologos de la ciencia. ${ }^{61}$ 


\section{NOTAS}

1. En la polémica están presentes otros debates que de una manera $u$ otra inciden en la cues. tión. Por un lado, el que se da en el seno de la misma sociología en relación a su estatus cientufico y su capacidad explicativa (causal, intencional. ftncional); por otro, el que tiene lugar en la psicología en tomo a las posiciones cognitivas eliminativistas y las que dejan espacio a la intencionaldad y la racionalidad; finalmente el que tiene que ver con la naturalización de la racionalidad.

2. La distinción externolintemo no colncide con la que se establece entre contexto de descubrimiento $y$ de justificacion, ya que el primero puede entenderse en términos de factores internos, mientras que alos contenidos cientificos? lo son en téminos de factores externos según los sociólogos.

3. Merton (1938). Esta separación la acepta incluso en $1933 \mathrm{cn}$ su epoca más radical.

4. Mannheim (1929).

5. Esto es debido a la universalidad de las afirmaciones y aplicaciones de las ciencias naturales. su recurso a pruebas y demostraciones, sus componentes formales seguin Mannheim (1929).

6. Los primeros trabajos de Kuhn son, sin embargo, una fuente de inspiración para los sociólogos. La otra influencia, reconocida explicitamente, es la de los trabajos matxistas sobre la ciencia. No es casualidad la enome presencia de autores de los paises del Este en la sociología del conocimiento (húngaros, yugoslavos, alemanes del este). Ver I. Hronszky y M. Feher (1988).

7. Bloor (1976: 4-5) define este principio: Jos mismos tipos de causas han de explicar las creencias falsas y las verdaderas.

8. Bames (1977; 8).

9. Bames (1974, 1977).

10. Bloor (1976).

11. Por ejemplo en Popper (1979, 1982a, 1982b).

12. Popper (1994).

13. Popper (1994; 102).

14. Popper (1994; 101) afirma: wa sociologia del conocimiento pasa por alto a la propia sociologia del conocimiento - la teoria de la objetividad científica - que sollo puede explicarse en tér. minos de ideas sociales como la competencia de la tradición crítica de las instituciones, del poder del estador.

15. Por ejemplo en Lakatos (1974 o 1983).

16. Chalmers (1992: 122) esta de acuerdo con Lakatos y afima sque existe un dominio legítimo para la historia intema y otro para la sociología.

17. Lakatos (1984) admite como interna la influencia de factores como los culturales o religiosos tradicionalmente tratados como extemos: afirma (1984: 14): wun puritano como tal tenía buenas razones para adoptar una aproximacion instrumental empiristam.
18. En Lakatos (1974: 13) o en Lakatos y Musgrave (1974: 239).

19. D. Bloor (1988) critica la postura de Lakatos y resalta esa dualidad.

20. N. Koertge crilica a Lakatos, pero desde una perspectiva más bien de corte popperiano en la que las teorias se miden por su capacidad de resolver problemas y donde los casos en contra pueden cuestionarla. Mantiene restos del método critico y cuestiona los cinturones de protección lakatosonianos. En Lakatos (1974a: 121-138).

21. Laudan (1986).

22. Dìstinguiendo tambiên muy bien el sociologismo (que relaciona con *algunos marxismos") que reduce toda creencia a causación social y el idealismo de corte hegeliano que niega que haya habido alguna transfomacion historica de las ideas debidas a cambios en las estructuras sociales, Laudan (1986: 248).

23. Laudan (1986: 251 ).

24. Laudan (1986: 252). Esto se basa en su aceptación del usupuesto de arracionalidad" que propone Merton.

25. Laudan (1986: 261).

26. Laudar (1986: 252). Bames (1977: 28) viene aquí en apoyo de Laudan cuando afirma que la sociología señala condiciones necesarias pero no suficientes de la producción de conocimiento. por tanto deja espacio para la epistemología $y$ cuestiones como la contrastabilidad.

27. Para Laudan (1986) sracionalidad cientfficax es seguir el método cientílico. R.E. Butts (1984: 239) en su trabajo sobre Kant, está de acuerdo con Laudan en insistir en que "las explicaciones de las creencias cientificas deben conformarse a alguna teoria de la racionalidad (co. rrecta)\%. Esto es posible desde algún tipo de "meta-razones" metodologicas como la resolución de problemas.

28. I. Hacking (1981: 268-269) critica a Lakatos por dejar demasiada historia de la ciencia real abandonada a lo irracional y la explicacion externa.

29. J. Agassi e I. Jarvie (1987).

30. Wettersten (1987).

31. Kekes (1987).

32. I.C. Jarvie (1984a), con Agassi (1987). En (1984b: 176.177) debate directamente con el Strong Programme y acusa a Bloor y Laudan de cometer el error de entender a la ciencia en términos de las acreencias" de los científicos en lil. gar de hacerlo en términos de sus kafimaciones» objetivas en el sentido de Popper. Chalmers (1981: 17, 1992: 115) considera de acuerdo con Popper que "las creencias de los individuos son bastante inapropiadas para comprender la naturaleza de la ciencia y su progreson.

33. J. Agassi y R. Wettersten (1987: 295) afir- 
man: "[...] nosotros podemos encontrar un incremento en la profundidad y en la elección de problemas y los lectores pueden realnente comprobar, sea lo que sea esta desiderata, si los subrayados aquí u otros, son cuizds mejores*.

34. Este ntevo modelo proporciona constricciones débiles en la selección de los objetivos cognoscitivos de las ciencias, sin embargo el cornponente normativo no desaparece totalmente ya que no sólo describe la forma en que se desa. rrollan los valores cognitivos en la ciencia síno que también trata de ofrecer una caracterización normativamente viable del modo en que tendria que desarrollarse la discusion sobre tales valores. Los objetivos o metas cognitivas son, para Lau. dan, susceptibles de discusion racional al contrario de la tesis de Kuhn, Popper o Reichenbach.

35. Cumpliendo el requerimiento de simetría de Bloor (1976; 45).

36. P. Suppes (1984).

37. Elster $(1988,1989,1990)$ por ejemplo. Esta posición es compartida en genernl por marxistas analíticos y por teóricos de la acción y elección colectiva.

38. C. Chemiak (1986) apoyándose en la cognitiva, Ver también S. Fuller (1988, 1989).

39. La propuesta de H.A. Simon y D. Kulkarni va en esta dirección en relación al cambio de creencias y por tanto al de la elección entre teoras cientificas. H.A. Simon (1989); Simon y D. Kulkami (1988) tratan la racionalidad en términos cognitivos e informacionales, pero tambicn en teminos de la teoria de la decisión. Las nociones de Simon son racionalidad urestringidas o *acotada. Su teoria de la "satisfacción* ha tenido amplia aceptacion. No hay que olvidar tampoco los esfuerzos por caracterizar la racionalidad $y$ actitudes episténicas en relación al conocimiento científico de autores corno R. Foley (1987) o Harsing (1982).

40. Newton-Smith (1987). J.R. Brown (1984:7) mantiene de acuerdo con Kuhn, que las ciencias naturales no necesitan una explicación sociologica sino una nueva noción de racionalidad que no ofrezca una vision acumulativa de la historia de la ciencia. Entiende la racionalidad nomativa de Laudan, por ejemplo, como un ideal regulativo. E. McMullin (1984: 136) es claro al respecto, Ia racionalidad de la ciencia se puede construir de dos formas, una preguntando por la racionalidad del cientifico (subjetiva), que normas realmente guian su investigacion, la otra desde una ractonalidad normativa desde el presente. La realmente explicativa es la primera aunque la segunda permita construir modelos evaluntivos para dar cuenta por ejemplo del progreso. G. Gutting (1984: 97) dialoga con los sociologos proponiendo un acuerdo en tomo a la explicación racional débil. El so. ciólogo de Gutting admite este tipo de explicación aunque prefiere la causal.
41. Esto no excluye la legitimidad de la pregunta por la racionalidad de los fines de interés en el contexto de otras teorias de la racionalidad. Desde la propuesta que estamos examinando lo relevante es explicar por qué los sujetos mantienen ciertas creencias, realizan ciertos actos o llevan a cabo determinadas decisiones o elecciones, en relación a ciertos objetivos a metas. La racionalidad de esos objetivos y los criterios a seguir es una cuestión dependiente de la concepción de la ciencia dominante en cada momento, y puede estar además relacionada con factores de tipo social, politico o ético, no solamente epistémicos.

42. Newton-Smith (1987), A. Gomez (1991).

43. Ambas en Elster (1989).

44. Excluye su consideración como ley de cobertura, Nosotmos usaremos el término *racionalidad minimay siguiendo la terminologia de Newton-Smith (1981) o Cherniak (1986).

45. Como afiman por ejemplo Newton-Smith (1987: 268) y G. Gutting (1984) en J.R. Brown (1984).

46. La sociologia podria tal vez explicar cómo se producen sociologicamente ciertos criterios epistemológicos o metodológicos, lo cual sería una cuestión diferente. Pues ha de admitir que las creencias que se mantienen dependen de la relación evaluativa que con tales criterios internos mantienen los sujetos como afima el racionalista.

47. Bloor (1976: 2). En este sentido Jarvie (1984: 179) considera a Bloor un "positivista e inductivista ingenno*.

48. Bames (1974: 25$)$.

49. Newton-Smith (1987:272).

50. Para Newton-Smith (1987: 269) una sociologia científica del conocimiento ha de compromenterse con el uso de las categorias de verdad y racionalidad,

51. Como afima L.C. Jarvie (1984: 60-61) "Se pueden mantener racionalmente creencias falsas.

52. Según I.C. Janvie (1984: 61) ]a racionalidad no tiene que ver con la verdad de las creencias. Como muestra J. Elster a lo largo de su obra, ver por ejemplo (1984, 1989, 1990).

53. La evaluación de la situación pocas veces se hace de forma máximamente racional de acuerdo con los modelos nommativos: desde creencias objetivamente racionales, atendiendo a toda la información disponible y según un calculo optimo de lo que es objetivamente adectado en la situación. Habitualmente la evaluación se efectua desde creencias que no son las más adecuadas o incluso erróneas o falsas, desde el punto de vista de un modelo normativo, desde principios y objetivos, que conducen a decisiones y acciones to optimas $\mathrm{u}$ objetivamente inadecuadas según tal modelo.

54. Evidentemente, ello no excluye que las per- 
sonas puedan actuar a veces inconsistentemente, en contra de lo que han decidido ( $\alpha$ Akrasian, udebilidad de la voluntad, son formas de hacer inteligible este hecho).

55. Ver en este sentido a Bartley (1987: 443450), en el que señala la necesidad de estudiar la comunidad cientrfica desde teorías como la de la acción y elección colectiva dando importancia a los efectos no intencionales de la acción colectiva.

56. L. Harsing (1982) resalta en relacion al conocimiento cientifico la importancia de la discusión científica y su dimcnsión pública y, por tanto, la relevancia de la argumentación, la persuasión. los acuerdos y desacuerdos que genera, las condiciones que hacen posible el diálogo entre los científicos, las distorsiones a que está sometido éste y la configuración de actitudes. Ver también, en relación a este punto $\mathrm{S}$. Fuller (1993), y A.G. Gross (1990). Este último resalta la naturaleza retorica de la ciencia y el elemento consensual de la comunidad cientifica que el refiere directamente a los analisis de Habermas.

57. En la constitución de las creencias cientificas pueden influir factores externos, pero esta infuencia generalmente se entremezcla con la derivada de creencias cientificas dominantes en el momento y además la aceptnción de tales creen. cias incluye la evaluación diferencial según critetios internos a la ciencia. La misma ciencia tiene además mecanismos intemos que en el proceso de clesarrollo y evolución de una teoría permiten if expurgando la influencia externa, social, idco* lögica, etc. Esto ocurre, por ejemplo, en la constitucion y evolución de ciertas creencias cientificas del $x i x$ acerca de la inferioridad de las mujeres en terminos de la craneología o la teotía de la evolución.

58. La que no excluye la importancia de factores externos, por ejemplo, en relación a la discu- sión pública de ciertos objetivos relacionados con la aplicación de la ciencia y su repercusión sobre el medio social y natural, que puede incluir cuestiones de tipo político, económico, social o moral.

59. Lu que no impide la consideración de este lipo de explicación en términos causales entendiendo creencias, deseos y praferencias como antecedentes de la accion. El debate en torno a la naturaleza causal de entidades como éstas es clasico y sigue presente en la filosofia de la mente 0 de la accion.

60. Esto tiene que ver con la forma de entender la relación entre medio social y constitucion de creencias. Se rechaza el automatismo entre contexto social y creencias, los sujetos tienen un papel activo en la adquísición de creencias como muestran teorias de la acción y teorias cognitivas. En el caso de las creencias científleas, por más influencia que tenga el medio social en su aceptación ésta incluye los procesos de evaluacion de las sujetos. El proceso es enormemente complejo y el tipo de causalidad que opera en ellos exige mucho más que una caracterizacion mecanicista, a modo del Strong Programme, puesto que incluye cuestiones como la del significado y la intencionalidad.

61. La psicologfa social del conocimiento introduce un enfoque particular en el análisís de algunas de las cuestiones que se debaten en esta controversia. A través de tal psicologia pueden trazarse puentes entre las versiones más matizadas del racionalismo y la sociología cognitiva. En relación a esto es interesante la cercanfa de las tesis de la psicología con los supuestos que maneja la teoria de la racionalidad mínima, en relacion a la interpretación subjetiva de los contcxtos de actuacion, experiencia y conocimiento. Ver al respecto Daniel Bar-Tal y Yoram Bar-Tal (1988: 92).

\section{BIBLIOGRAFIA}

Agassi, Js, e I.Ch. Jarvie (eds.) (1987): Rationality: The Critical View, Dordrecht. M. Nijhoff.

Bar-TAl, D., y A.W. KRUGLANSKi (eds.) (1988): The social psychology of knowledge, Cambridge, Cambridge University Press.

BaRnes, B. (1974): Scientific Knowledge and Sociological Theory, Londres, Routledge \& Kegan Paul.

- (1977): Interesis and the Growth of Knowledye, Londres, Routledge \& Kegan Paul.

Bartiey, W.W. (1987): *Alienation Alienated: The Economics of Knowledge versus the Psycho. logy and Sociology of Knowledgen, en G. Radnitzky y W.W. Bartley, Evolutiontary Epistemology, Rationality, and the Sociology of Know ledge, La Salle, IL, Open Court.
BLoor, D. (1976): Knowledgre and Sacial Inaginewy, Londres, Routledge \& Kegan Paul.

- (1988); kationalism. Supernaturalism, and the Sociology of Knowledgew, en 1. Hronszky, M. Feher y B. Dajka (cds.) (1984), Scientific Knowledge Socialized, Dordrecht, Kluwer Academic.

Browm, J.R. (ed.) (1984): Scientific Rationality: The Sociological Tum, Dordrecht, D. Reidel.

Butrs, R.E. (1984): "The Role of Arational Factors in Interpretative History: The Case of Kant and ESP, en J,R, Browm (ed.) (1984).

Chalmers, A. (1992): La ciencia y como se elabora. Madrid, Siglo XXI.

ChernIAK, C. (1986): Minintal Rationality, Cambridge, MA, MIT. 
Euster, J. (1988): Uvas amaratas, Barcelona, Peninsula.

- (1989): Ulises y lar sirenas, México, FCE.

- (1990): Tuercas y tonillos. Una introducción a los conceptos básicos de las cientctas sociates, Barcelona, Gedisa.

FeYerabend, P.K. (1974): Contra el método, Barcelona, Arjel.

FOLEY, R. (1987): The Theory of Epistenologic Rationality, Cambridge, MA, Harvard University Press.

FuLLER, S, (1988); Social Epistentology, Bloomington, IN, Indiana University Press, 1991.

- (1989): Philosophy of Science and Its Discontents, Londres, Westwiew Press.

- (1993): Philosophy, Rhetoric, and the End of Knowledge, Madison, WI, University of Wisconsin Press.

Gomez, A. (1991): Sobre actores y tranoyas, Una explicacion situacional de la accion individual, Barcelona, Anthropos,

Gómez, R.J. (1993): "Kuhn y la racionalidad cien. tífica. ¿Hacia un kantianismo post-darwiniano?", en $O$. Nudler y G. Klimovsky, La racionalidad en debate. L. Racionalidad y conocimiento; la ractonalidad en la ciencia. Buenos Aires, Centro Editor de América Latina.

Gross, A.G. (1990): The Rhetoric of Science, Cambridge, MA, Harvard University Press.

Gurrive, G. (1984): *The Strong Program*, en J.R. Browm (ed.) (1984).

HaCkJng, I. (1985): Revoluciones cientificas, México, FCE.

HaRSTNo, L. (1982): Scientific Reasoning and Epistemic Aftitudes, Budapest, Akadémial Kiado.

JARve, I.C. (1984a): Rationality and Relativism, Londres, Routledge \& Kegan Paul.

- (1984b): «A Plague of Both Your Houses", en J.R. Browm (ed.) (1984).

KeKES, J. (1987): «Rationality and Problem-Solv. ing», en J. Agassi e I.Ch. Jarvie (eds.) (1987).

Koertae, N. (1974): *La critica inter-teórica y el desarrollo de la ciencias, en I. Lakalos (1974).
KunN, T.S. (1971): La estructura de las revoluciones cientificas, México, FCE.

LaKaTos, 1. (1974): Historia de la ciencia y sus recontstrucciones racionales, Madrid, Tecnos.

- (1983): La metodologia de los programas de investigación cientifica, Madrid, Alianza.

- y A. Muscrave (1974): La critica y el desarrollo del conocintento, Barcelona, Grijalbo.

LALDAN, L. (1986): El progreso y sus problemas. Hacia una teorla del crecimiento cientifico, Madrid, Encuentro.

MCMullin, E. (1984): "The Rational and the Social in the History of Sciencex, en J.R. Browm (ed.) (1984).

Mannhem, K (1929): Ideologia y utopta, Madrid, Aguilar, 1958.

Merton, R.K. (1938): Ciencia, tecnologia y sociedad en la Inglaterra del siglo XVI, Madrid, Alianza, 1984.

NewToN-Sмгтн, W.H. (1987): La racionalidad de la ciencia, Barcelona, Paidós.

Popper, K.R. (1979): El desarrollo del conocimiento cientifico, Buenos Aires, Paidos.

- (1982a); La lógica de la investigación cientifica, Madrid, Tecnos.

- (1982b): Contocintiento objetivo, Madrid, Tecnos.

- (1985): Reatismo y at objetivo de la ciencia, Madrid, Tecnos.

- (1994): En busca de un mutdo nejor, Barcelona, Paidós.

Simon, H.A. (1989): "The Scientist as Problem Salver», en D. Klahr y K. Kotovsky (eds.) (1989), Complex Infomtation Processing; Hillsdale, NJ, L, Erlbaum.

-, y D. Kulkarni (1988); «The Processes of Scientific Discovery: The Strategy of Experimentation $*$, Cognitive Science, 12.

Suppes, P. (1984): Probabilistic Metaphysic, Oxford, Blackwell.

Wettersten, J,R., y J. AGassi (1987): "The Choice of Problems and the Limits of Reason*, en J. Agassi e I.Ch. Jarvie (eds.) (1987). 